Pacific Journal of Mathematic 


\title{
ASYMPTOTIC CENTERS AND NONEXPANSIVE MAPPINGS IN CONJUGATE BANACH SPACES
}

\author{
TeCK-CheONG LiM
}

\begin{abstract}
This paper concerns fixed point theorems for nonexpansive mappings in conjugate Banach spaces. An example shows that there exist fixed-point-free affine isometries on weak* compact convex sets. Asymptotic centers of decreasing net of founded sets in $l^{1}$ are shown to be compact and a common fixed point theorem for left reversible topological semigroup of nonexpansive mappings in $l^{1}$ is given.
\end{abstract}

1. Introduction. Let $K$ be a nonempty weakly compact convex subset of a Banach space and $T: K \rightarrow K$ a nonexpansive mapping, i.e., $\|T x-T y\| \leqq\|x-y\|, x, y \in K$. A theorem of Kirk [10] (see also Browder [1], Gödhe [6]) states that if $K$ has normal structure then $T$ has a fixed point. Whether the condition of normal structure is essential remains an open problem, although Schöneberg [13] has shown that some weakenings of normal structure suffice. With a slight modification of normal structure, Kirk's proof of his theorem also yields the following theorem in conjugate Banach spaces.

THEOREM 1 (Kirk). Let $K$ be a nonempty weak* compact convex subset of a conjugate Banach space and assume that $K$ possesses weak* normal structure (see Definition 1 in §3). Then every nonexpansive selfmapping of $K$ has a fixed point.

One major observation presented in this note is that the condition of weak* normal structure in Theorem 1 is essential, even for affine isometries. We also derive a sufficient condition for a conjugate Banach space to have weak* normal structure. In particular, we show that $l_{1}$ possesses weak* normal structure. Asymptotic centers of decreasing nets of bounded subsets in $l_{1}$ are shown to form a normcompact nonempty subset and an application of this result is made to obtain a common fixed point theorem for families of nonexpansive mappings in $l_{1}$.

2. A counterexample. Let $c_{0}$ be the space of null sequences, equipped with the sup norm \|\|$_{\infty},\|x\|_{\infty}=\sup _{i \geq 1}\left|x_{i}\right|$, and $l_{1}$ the space of absolutely summable sequences equipped with the norm \|\|$_{1},\|x\|_{1}=\sum_{i=1}^{\infty}\left|x_{i}\right|$. For each sequence $x$, let $x^{+}$and $x^{-}$be the positive and negative part of $x$, respectively. Renorm $c_{0}$ by the 
new norm defined by

$$
|x|=\left\|x^{+}\right\|_{\infty}+\|x-\|_{\infty} .
$$

$|\cdot|$ is equivalent to $\|\cdot\|_{\infty}$ since $\|x\|_{\infty} \leqq|x| \leqq 2\|x\|_{\infty}$. This method of renorming was used by Bynum [4] to renorm $l_{p}, 1<p<\infty$.

LEMma 1. The dual of $\left(c_{0},|\cdot|\right)$ is isometrically isomorphic to $\left(l_{1},\|\cdot\|\right)$ with the norm $\|\cdot\|$ defined by

$$
\|x\|=\max \left(\left\|x^{+}\right\|_{1},\left\|x^{-}\right\|_{1}\right) .
$$

Proof. Since $|\cdot|$ is equivalent to $\|\cdot\|_{\infty}$, the dual of $\left(c_{0},|\cdot|\right)$ is representable by $l_{1}$. It suffices to show that

$$
\max \left(\left\|f^{+}\right\|_{1},\left\|f^{-}\right\|_{1}\right)=\sup \left\{\sum_{i=1}^{\infty} x_{i} f_{i}: x \in c_{0},\left\|x^{+}\right\|_{\infty}+\left\|x^{-}\right\|_{\infty} \leqq 1\right\}
$$

for each $f=\left(f_{i}\right) \in l_{1}$. Note that the supremum on the right can be taken over $x$ satisfying the further requirment that $x_{i} f_{i} \geqq 0$ for all $i$. (If $x_{i} f_{i}<0$, replace $x$ by another one with $x_{i}=0$.) It then follows that

$$
\begin{aligned}
\sum_{i=1}^{\infty} x_{i} f_{i} & \leqq\left\|x^{+}\right\|\left\|_{\infty}\right\| f^{+}\left\|_{1}+\right\| x^{-}\left\|_{\infty}\right\| f^{-} \|_{1} \\
& \leqq \max \left(\left\|f^{+}\right\|_{1},\left\|f^{-}\right\|_{1}\right) .
\end{aligned}
$$

For the reverse inequality, note that one can approximate $\left\|f^{+}\right\|_{1}$ $\left(\left\|f^{-}\right\|_{1}\right)$ by $\sum_{i=1}^{\infty} x_{i} f_{i}$ by suitably choosing $x_{i}=1$ or 0 ( -1 or 0 ).

ExAmple 1. Let $K=\left\{\left(x_{i}\right) \in l_{1}: x_{i} \geqq 0, \sum_{i=1}^{\infty} x_{i} \leqq 1\right\} . \quad K$ is a weak* compact convex set in $\left(l_{1},\|\cdot\|\right)$ since it is the intersection of the unit ball and the weak ${ }^{*}$ closed set $\left\{\left(x_{i}\right): x_{i} \geqq 0\right\}$. Let $T: K \rightarrow K$ be the mapping defined by the equation

$$
T x=\left(1-\sum_{i=1}^{\infty} x_{i}, x_{1}, x_{2}, \cdots, x_{n}, \cdots\right)
$$

for $x=\left(x_{i}\right) \in K$. We show that $T$ is an isometry. Let $x, y \in K$ and let $I=\left\{i \in \boldsymbol{Z}^{+}: x_{i}-y_{i} \geqq 0\right\}$ and $J=\left\{j \in \boldsymbol{Z}^{+}: x_{j}-y_{j}<0\right\}$. Assume that $\sum_{i \in I} x_{i}-y_{i} \geqq \sum_{j \in J} y_{j}-x_{j}$. Then $\|x-y\|=\sum_{i \in I} x_{i}-y_{i}$ and

$$
\begin{aligned}
\|T x-T y\| & =\left\|\sum_{i=1}^{\infty}\left(y_{i}-x_{i}\right), x_{1}-y_{1}, \cdots, x_{n}-y_{n}, \cdots\right\| \\
& =\left\|\sum_{j \in J}\left(y_{j}-x_{j}\right)-\sum_{i \in I}\left(x_{i}-y_{i}\right), x_{1}-y_{1}, \cdots, x_{n}-y_{n}, \cdots\right\| \\
& =\max \left(\sum_{i \in I} x_{i}-y_{i}, \sum_{i \in I} x_{i}-y_{i}\right) \\
& =\sum_{i \in I} x_{i}-y_{i}=\|x-y\| .
\end{aligned}
$$


Similarly, we also have $\|T x-T y\|=\|x-y\|$ in case $\sum_{i \in I} x_{i}-y_{i} \leqq$ $\sum_{j \in J} y_{j}-x_{j}$. Hence $T$ is an isometry. $T$ is clearly affine and fixed point free. Further properties of $K$ and $T$ are listed in the following:

(1) $\lim \left\|y-T^{n} x\right\|=\operatorname{Diam}(K)=1, y, x \in K$.

(2) $K$ does not possess weak* normal structure. This is necessarily true by Theorem 1 and the above demonstration.

(3) $T^{n} x$ converges weakly* to zero for each $x \in K$.

(4) $K$ itself is a minimal $T$-invariant weak* compact convex set. Indeed every $T$-invariant weak* comyact convex subset $C$ of $K$ must contain 0 by (3). Hence $T^{n}(0)=e_{n} \in C$ for all $n$. Therefore $K=$ $\overline{\mathrm{Co}}\left(\left\{e_{n}\right\} \cup\{0\}\right) \subseteq C$ and $C=K$.

The above example shows that the condition of weak* normal structure cannot be removed from Theorem 1 even if the nonexpansive mapping is an affine isometry. In contrast, every affine nonexpansive selfmapping of a weakly compact convex set always has a fixed point.

3. Conjugate Banach spaces having weak normal structure. In this section we derive a condition for a conjugate Banach space to have weak* normal structure.

Definition 1. A weak* closed convex subset $C$ of a conjugate Banach space is said to have weak* normal structure if every weak* compact convex subset $K$ of $C$ containing more than one point contains a point $x_{0}$ such that

$$
\sup \left\{\left\|x_{0}-y\right\|: y \in K\right\}<\operatorname{diam} K .
$$

In the following theorem, $\boldsymbol{R}^{+}=\{\boldsymbol{r} \in \boldsymbol{R}: \boldsymbol{r} \geqq 0\}$ and the notation $x_{n} \stackrel{*}{\rightarrow} y$ will denote the weak* convergence of $x_{n}$ to $y$.

THEOREm 2. Let $X$ be a the conjugate space of a separable Banach space. Suppose that there exists a function $\delta: \boldsymbol{R}^{+} \times \boldsymbol{R}^{+} \rightarrow \boldsymbol{R}^{+}$ satisfying the following conditions.

(i) For each fixed $s, \delta(r, s)$ is continuous and strictly increasing in $r$,

(ii) $\delta(s, s)>s$ for every $s>0$,

(iii) if $x_{n} \stackrel{*}{\rightarrow} 0$ and $\lim \left\|x_{n}\right\|=s>0$, then

$$
\lim \left\|y-x_{n}\right\|=\delta(\|y\|, s) \text { for every } y \in K \text {. }
$$

Then every weak* closed convex subset of $X$ has weak* normal structure. 
Proof. Suppose on the contrary that $X$ contains a weak* closed convex subset $C$ which does not have weak* normal structure. Then there exists a weak* compact convex subset $K$ of $C$ with Card $K>1$ and for every $x \in K$

$$
\sup \{\|x-y\|: y \in K\}=\operatorname{diam} K=d>0 .
$$

By a method of Brodskii-Milman [3], there exists a sequence $\left\{x_{n}\right\} \subset K$ such that $\lim d\left(x_{n+1}, \mathrm{Co}\left(x_{i}\right)_{i \leq n}\right)=d$. Since subsequences of $\left\{x_{n}\right\}$ share the same property, we may assume that $x_{n} \stackrel{*}{\rightarrow} x_{0}$ for some $x_{0} \in K$ and $\lim \left\|x_{n}-x_{0}\right\|=s$. Clearly, $s>0$. For each fixed $m$, we have $\lim _{n}\left\|x_{m}-x_{n}\right\|=d$. Therefore, by (iii)

$$
\mathrm{d}=\lim _{n}\left\|\left(x_{n}-x_{0}\right)-\left(x_{m}-x_{0}\right)\right\|=\delta\left(\left\|x_{m}-x_{0}\right\|, s\right) .
$$

Using (i), $d=\delta(s, s)$. Using (ii), we have $s<d$. We shall show that $\sup \left\{\left\|x_{0}-y\right\|: y \in K\right\} \leqq s$. Suppose not, then there exists $z \in K$ with $\left\|z-x_{0}\right\|>s$. Then

$$
\begin{aligned}
\lim \left\|z-x_{n}\right\| & =\lim \left\|\left(z-x_{0}\right)-\left(x_{n}-x_{0}\right)\right\| \\
& =\delta\left(\left\|z-x_{0}\right\|, s\right) \\
& >\delta(s, s)=d
\end{aligned}
$$

by (iii) and (i). This is impossible. Therefore, $\sup \left\{\left\|x_{0}-y\right\|: y \in K\right\} \leqq$ $s<d$, which again contradicts our initial assumption. Hence $C$ has weak* normal structure.

The next proposition shows that the spaces $l_{p}, p \geqq 1$ satisfy the condition in Theorem 2 with $\delta(r, s)=\left(r^{p}+s^{p}\right)^{1 / p}$.

Proposition 1. In $l_{p}$, if $x_{n} \stackrel{*}{\rightarrow} x$, then for every $y \in l_{p}$,

$$
\lim \sup \left\|x_{n}-y\right\|^{p}=\lim \sup \left\|x_{n}-x\right\|^{p}+\|x-y\|^{p} .
$$

In particular, if $\lim \left\|x_{n}-x\right\|$ exists, we have

$$
\lim \left\|x_{n}-y\right\|=\left(\lim \left\|x_{n}-x\right\|^{p}+\|x-y\|^{p}\right)^{1 / p} .
$$

Proof. For $p=1$, the equality is a special case of a more general equality given in Proposition 2; see Corollary 3. For $p>1$, let $J: l_{p} \rightarrow l_{q}, 1 / q+1 / p=1$, be the duality mapping defined by

$$
J x=\left(\left|x_{1}\right|^{p-1} \operatorname{sgn} x_{1}, \cdots,\left|x_{n}\right|^{p-1} \operatorname{sgn} x_{n}, \cdots\right) .
$$

$J$ is weakly continuous and $\langle J x, x\rangle=\|x\|^{p}$, see [2]. Since $J$ is the subdifferential of the convex function $f(x)=1 / p\|x\|^{p}$, we have

$$
\frac{1}{p}\left\|x_{n}-y\right\|^{p}=\frac{1}{p}\left\|x_{n}-x\right\|^{p}+\int_{0}^{1}\left\langle J\left(x_{n}-x+t(x-y), x-y\right)\right\rangle d t
$$


(Gossez-Lami-Dozo [7]). Therefore

$$
\begin{aligned}
\lim \sup \left\|x_{n}-y\right\|^{p} & =\lim \sup \left\|x_{n}-x\right\|^{p}+p \int_{0}^{1} t^{p-1}\|x-y\|^{p} d t \\
& =\lim \sup \left\|x_{n}-x\right\|^{p}+\|x-y\|^{p} .
\end{aligned}
$$

Proposition 1 and Theorem 2 implies that every weak* closed convex subset of $l_{1}$ has weak* normal structure. Note that such a set may not possess normal structure. For a simple example, let $C$ be the unit ball and $K=\left\{\left(x_{i}\right): x_{i} \geqq 0, \sum_{i=1}^{\infty} x_{i}=1\right\}$. Then $K$ is closed convex and $\sup \{\|x-y\|: y \in K\}=\operatorname{diam} K=2$ for every $x \in K$. Combining this result with Theorem 1 we have the following result of Karlovitz [9].

CoRollary 1 [9]. Let $K$ be a weak* compact convex nonempty subset of $l_{1}$ and $T: K \rightarrow K$ be a nonexpansive mapping. Then $T$ has a fixed point.

\section{Asymptotic centers in $l_{1}$.}

Definition 2 [12]. Let $C$ be a nonempty subset of a Banach space $X$ and $\left\{B_{\alpha}: \alpha \in \Lambda\right\}$ a decreasing net of bounded nonempty subsets of $X$. For each $x \in C$ and $\alpha \in \Lambda$, let

$$
\begin{aligned}
r_{\alpha}(x) & =\sup \left\{\|x-y\|: y \in B_{\alpha}\right\}, \\
r(x) & =\lim _{\alpha} r_{\alpha}(x)=\inf _{\alpha} r_{\alpha}(x),
\end{aligned}
$$

and

$$
r=\inf \{r(x): x \in C\} \text {. }
$$

The set (possibly empty) $\mathscr{C} \mathscr{C}\left(\left\{B_{\alpha}: \alpha \in \Lambda\right\}, C\right)=\{x \in C: r(x)=r\}$ and the number $r$ will be called, respectively, the asymptotic center of $\left\{B_{\alpha}: \alpha \in \Lambda\right\}$ w.r.t. $C$ and the asymptotic radius of $\left\{B_{\alpha}: \alpha \in \Lambda\right\}$ w.r.t. $C$.

Proposition 2. Let $\left\{B_{\alpha}: \alpha \in \Lambda\right\}$ be a decreasing net of bounded subsets of $l_{1}$ and $y_{n}$ a weak* convergent sequence with weak* limit $y$. Then

$$
\begin{array}{r}
\lim _{\alpha} \sup \left\{\|y-x\|: x \in B_{\alpha}\right\}+\limsup _{n} \sup _{n}-y \| \\
=\limsup _{n} \limsup _{\alpha}\left\{\left\|y_{n}-x\right\|: x \in B_{\alpha}\right\} .
\end{array}
$$

Proof. For $x \in l_{1}$, we shall denote by $x^{(i)}$ the $i$ th coordinate of $x$.

By the triangle inequality, we clearly have the inequality $\geqq$ in (2). By a simple diagonal process, we may assume that $\left\{B_{\alpha}: \alpha \in \Lambda\right\}$ 
is a decreasing sequence $\left\{B_{n}: n \geqq 1\right\}$ of bounded sets. Choose $x_{n} \in B_{n}$ such that $\lim \sup \left\|y-x_{n}\right\|=\lim \sup _{n}\left\{\|y-x\|: x \in B_{n}\right\}$. It follows that it suffices to prove the following inequality:

$$
\lim \sup _{n}\left\|y-x_{n}\right\|+\lim \sup _{m}\left\|y_{m}-y\right\| \leqq \lim \sup _{m} \lim \sup _{n}\left\|y_{m}-x_{n}\right\| \text {. }
$$

We may also assume, without loss of generality, that $y=0$, and that $\lim \left\|x_{n}\right\|, \lim \left\|y_{m}\right\|$, and $\lim _{m} \lim \sup _{n}\left\|y_{m}-x_{n}\right\|$ exist.

Let $r=\lim _{m} \lim \sup _{n}\left\|y_{m}-x_{n}\right\|$ and $k=\lim \left\|y_{m}\right\|$. Suppose, on the contrary that $\lim \left\|x_{n}\right\|=r-k+p$ for some $p>0$. Let $p>$ $\varepsilon>0$. Let $m_{1}, N_{1}$ and $M_{1}\left(N_{1}\right.$ and $M_{1}$ depend on $\left.m_{1}\right)$ be sufficiently large integers such that

$$
\begin{aligned}
& || y_{m_{1}} \| \geqq k-\frac{\varepsilon}{4}, \\
& \sum_{N_{1}+1}^{\infty}\left|y_{m_{1}}^{(i)}\right| \leqq \frac{\varepsilon}{8} \\
& \left\|x_{n}-y_{m_{1}}\right\| \leqq r+\frac{\varepsilon}{4},
\end{aligned}
$$

and

$$
\left\|x_{n}\right\| \geqq r-k+p-\frac{\varepsilon}{4}, \text { for all } n \geqq M_{1} .
$$

Then for $n \geqq M_{1}$, we have

$$
\begin{aligned}
r+\frac{\varepsilon}{4} \geqq\left\|x_{n}-y_{m_{1}}\right\| & =\sum_{1}^{N_{1}}\left|x_{n}^{(i)}-y_{m_{1}}^{(i)}\right|+\sum_{N_{1}+1}^{\infty}\left|x_{n}^{(i)}-y_{m_{1}}^{(i)}\right| \\
& \geqq \sum_{1}^{N_{1}}\left|y_{m_{1}}^{(i)}\right|-\sum_{1}^{N_{1}}\left|x_{n}^{(i)}\right|+\sum_{N_{1}+1}^{\infty}\left|x_{n}^{(i)}\right|-\sum_{N_{1}+1}^{\infty}\left|y_{m_{1}}^{(i)}\right| \\
& =\left\|y_{m_{1}}\right\|-2 \sum_{N_{1}+1}^{\infty}\left|y_{m_{1}}^{(i)}\right|+|| x_{n} \|-2 \sum_{1}^{N_{1}}\left|x_{n}^{(i)}\right| \\
& \geqq k-\frac{\varepsilon}{4}-\frac{\varepsilon}{4}+r-k+p-\frac{\varepsilon}{4}-2 \sum_{1}^{N_{1}}\left|x_{n}^{(i)}\right| .
\end{aligned}
$$

Hence

$$
\sum_{1}^{N_{1}}\left|x_{n}^{(i)}\right| \geqq \frac{1}{2}(p-\varepsilon), n \geqq M_{1} .
$$

Since $y_{m} \stackrel{*}{\rightarrow} 0$ there exist $m_{2}, N_{2}>N_{1}$ and $M_{2}>M_{1}\left(N_{2}\right.$ and $M_{2}$ depend on $m_{2}$ ) such that

$$
\begin{aligned}
& \sum_{1}^{N_{1}}\left|y_{m_{2}}^{(i)}\right| \leqq \frac{\varepsilon}{10}, \\
& \left\|y_{m_{2}}\right\| \geqq k-\frac{\varepsilon}{5},
\end{aligned}
$$




$$
\begin{aligned}
& \sum_{N_{2}+1}^{\infty}\left|y_{m_{2}}^{(i)}\right| \leqq \frac{\varepsilon}{10} \\
& \left\|x_{n}-y_{m_{2}}\right\| \leqq r+\frac{\varepsilon}{5}
\end{aligned}
$$

and

$$
\left\|x_{n}\right\| \geqq r-k+p-\frac{\varepsilon}{5}, \text { for } n \geqq M_{2} \text {. }
$$

Then for $n \geqq M_{2}$, we have

$$
\begin{aligned}
& v+\frac{\varepsilon}{5} \geqq\left\|x_{n}-y_{m_{2}}\right\|=\sum_{1}^{N_{1}}\left|x_{n_{1}}^{(i)}-y_{m_{2}}^{(i)}\right|+\sum_{N_{1}+1}^{N_{2}}\left|x_{n}^{(i)}-y_{m_{2}}^{(i)}\right|+\sum_{N_{2}+1}^{\infty}\left|x_{n}^{(i)}-y_{m_{2}}^{(i)}\right| \\
& \geqq \sum_{1}^{N_{1}}\left|x_{n}^{(i)}\right|-\sum_{1}^{N_{1}}\left|y_{m_{2}}^{(i)}\right|+\sum_{N_{1}+1}^{N_{2}}\left|y_{m_{2}}^{(i)}\right|-\sum_{N_{1}+1}^{N_{2}}\left|x_{n}^{(i)}\right| \\
&+\sum_{N_{2}+1}^{\infty}\left|x_{n}^{(i)}\right|-\sum_{N_{2}+1}^{\infty}\left|y_{m_{2}}^{(i)}\right| \\
&=\left\|y_{m_{2}}\right\|-2 \sum_{1}^{N_{1}}\left|y_{m_{2}}^{(i)}\right|-2 \sum_{N_{2}+1}^{\infty}\left|y_{m_{2}}^{(i)}\right| \\
&+\left\|x_{n}\right\|-2 \sum_{N_{1}+1}^{N_{2}}\left|x_{n}^{(i)}\right| \\
& \geqq k-\frac{\varepsilon}{5}-\frac{\varepsilon}{5}-\frac{\varepsilon}{5}+r-k+p-\frac{\varepsilon}{5}-2 \sum_{N_{1}+1}^{N_{2}}\left|x_{n}^{(i)}\right| .
\end{aligned}
$$

Hence

$$
\sum_{N_{1}+1}^{N_{2}}\left|x_{n}^{(i)}\right| \geqq \frac{1}{2}(p-\varepsilon) \text { for } n \geqq M_{2} \text {. }
$$

Continuing in this way, we obtain two sequences $M_{1}<M_{2}<\ldots$ and $N_{1}<N_{2}<\cdots$ such that for $n \geqq M_{k}$,

$$
\sum_{N_{k-1}+1}^{N_{k}}\left|x_{n}^{(i)}\right| \geqq \frac{1}{2}(p-\varepsilon), \quad N_{0}=0 .
$$

Thus for $n \geqq M_{k},\left\|x_{n}\right\| \geqq \sum_{1}^{N_{k}}\left|x_{n}^{(i)}\right| \geqq k \cdot 1 / 2(p-\varepsilon)$. This contradicts the boundedness of the sequence $x_{n}$.

COROLlary 2. Let $x_{n}$ be a bounded sequence in $l_{1}$ and $y_{n} \stackrel{*}{\rightarrow} y$. Then $\lim \sup \left\|x_{n}-y\right\|+\lim \sup \left\|y_{m}-y\right\|=\lim \sup \lim \sup \left\|x_{n}-y_{m}\right\|$

Corollary 3. Proposition 1 for $p=1$.

THEOREM 3. Let $C$ be a weak* closed convex nonempty subset of $l_{1}$ and $\left\{B_{\alpha}: \alpha \in \Lambda\right\}$ a decreasing net of bounded nonempty subsets 
of C. Let the function $r(x)$ be defined as in Definition 2. Then for each $s \geqq 0,\{x \in C: r(x) \leqq s\}$ is weak* compact convex and the asymptotic center of $\left\{B_{\alpha}: \alpha \in \Lambda\right\}$ w.r.t. $C$ is a nonempty (norm) compact convex subset of $C$.

Proof. Let $K_{s}=\{x \in C: r(x) \leqq s\}$ and let $K$ be the asymptotic center. Clearly, $\operatorname{diam}\left(K_{s}\right) \leqq 2 s$. Since $r(\cdot)$ is a convex function, $K_{s}$ is also convex. To show that $K$ is weak* compact, it suffices to sohw that $K_{s}$ is weak* closed. Let $y_{n} \in K_{s}$ and $y_{n} \stackrel{*}{\rightarrow} y$. By Proposition 2.

$$
r(y)=\lim \sup r\left(y_{n}\right)-\lim \sup \left\|y_{n}-y\right\| \leqq s .
$$

Hence $y \in K_{s}$ and $K_{s}$ is weak* closed. Suppose now that $s=r$, where $r$ is the asymptotic radius of $\left\{B_{\alpha}: \alpha \in A\right\}$ w.r.t. $C$. If $r\left(y_{n}\right)=r$, then we must have $\lim \sup \left\|y_{n}-y\right\|=0$ for otherwise $r(y)<r$, a contradiction to the definition of $r$. Therefore, for a sequence in $K$, weak ${ }^{*}$ convergence implies norm convergence. Hence $K$ is compact. Since $K=\bigcap\left\{K_{s}: K_{s} \neq \varnothing\right\}$ and each $K_{s}$ is nonempty weak* compact, we have $K \neq \varnothing$.

Corollary 4. Let $C$ be a weak* closed convex subset of $l_{1}$ and $D$ a nonempty bounded subset of $C$. Then the Chebyshev center of $D$ w.r.t. $C$ is nonempty compact convex. In particular, for any two points $x$ and $y$, the set $\left\{z \in l_{1}:\|z-x\|=\|z-y\|=1 / 2\|x-y\|\right\}$ is compact.

Proof. If we let $B_{\alpha}=D$ for every $\alpha \in \Lambda$, the asymptotic center of $\left\{B_{\alpha}: \alpha \in \Lambda\right\}$ is the same as the Chebyshev center of $D$.

We conclude this section by giving an application of Theorem 3. Let $K$ be a set and $S$ a semigroup of selfmaps of $K$. $S$ is said to be a topological semigroup if $S$ is equipped with a Hausdorff topology such that for each $a \in S$, the two mappings from $S$ into $S$ defined by $s \rightarrow a s$ and $s \rightarrow s a$ for all $s \in S$, are continuous. $S$ is said to be left reversible if any two nonempty closed right ideals of $S$ have nonempty intersection (cf. [5, p. 34]). If $K$ is a topological space and $S$ a left reversible topological semigroup of selfmappings of $K$ suce that the mapping $(s, x) \rightarrow s(x)$ is separately continuous, then $S$ becomes a directed set if we define $a \geqq b$ if and only if $a S \subseteq \operatorname{cl}(b S)$. Moreover, if for a fixed element $u \in K$, we define $W_{s}=$ $\operatorname{cl}(s S(u)))$ for all $s \in S$, then the family $\left\{W_{s}: s \in S\right\}$ is a decreasing net of subsets of $K$ (see [8]). 
THEOREM 4. Let $C$ be a weak* closed convex nonempty subset of $l_{1}$ and $S$ a left reversible topological semigroup of nonexpansive selfmappings of $C$ such that the mapping $(s, x) \rightarrow s(x)$ is separately continuous. If for some $x \in C, s \in S, s S(x)$ is bounded, then $S$ has a common fixed point in $C$.

Proof. Let $W_{s}$ be defined as in the last paragraph. By Theorem 2 in [12], the asymptotic center $K$ of $\left\{W_{s}: s \in S\right\}$ is a $S$-invariant subset of $C$. By Theorem $4, K$ is a nonempty compact convev set. Since a compact convex set has normal structure, by Theorem 3 in [12] or Corollary 1 in [8], $S$ has a common fixed point in $K$.

\section{REFERENCES}

1. F. E. Browder, Nonexpansive nonlinear operators in a Banach space, Proc. Nat. Acad. U.S. A., 54 (1965), 1041-1044,

2. - Nonlinear functional analysis, Proc. Symp. Pure Math. vol. XVIII, Part 2, A. M. S., 1976.

3. M. S. Brodskii and D. P. Mil'man, On the center of a convex set, Doklady Alad. Nauk SSSR (N.S.), 59 (1948), 837-840.

4. W. L. Bynum, A class of spaces lacking normal structure, Compositio Math., 25 (1972), 233-236.

5. A. H. Clifford and G. B. Preston, The algebraitheory of semigroups, Vol. I, Math. Survey, no. 7. Amer. Math. Soc., Providence, R. I., 1961.

6. D. Gödhe, Zum prinzip der Kontraktiven Abbildung, Math. Nachr., 30 (1965), 251-258.

7. J. -P. Gossez and E. Lami Dozo, Some geometric properties related to the fixed point theory for nonexpansive mappings, Pacific J. Math., 40 (1972), 565-573.

8. R. D. Holmes and A. T. Lau, Nonexpansive actions of topological semigroups and fixed points, J. London Math. Soc., (2) 5 (1972), 330-336.

9. L. A. Karlovitz, On nonexpansive mappings, Proc. Amer. Math. Soc., 55 (1976), 321325.

10. W. A. Kirk, A fixed point theorem for mappings which do not increase distances, Amer. Math. Monthly, 72 (1965), 1004-1006.

11. T. C. Lim, A fixed point theorem for families of nonexpansive mappings, Pacific J. Math., 53 (1974), 487-493.

12. - Characterization of normal structure, Proc. Amer. Math. Soc., 43 (1974), 313-310.

13. R. Schöneberg, Private communication.

Received October 4, 1979 and in revised form November 2, 1979.

The University of Chicago

ChICAgo, IL 60637

Current address: Department of Mathematics George Mason University, Fairfax, VA 22030 



\section{PACIFIC JOURNAL OF MATHEMATICS}

\section{EDITORS}

DONALD BABBITT (Managing Editor)

University of Galifornia

Los Angeles, California 90024

HUGO RossI

University of Utah

Salt Lake City, UT 84112

C. C. MOORE AND ANDREW OGG

University of California

Berkeley, CA 94720
J. DUGUNDJI

Department of Mathematics University of Southern California Los Angeles, California 90007

R. FINN AND J. MILGRAM Stanford University

Stanford, California 94305

ASSOCIATE EDITORS

E. F. BECKENBACH

B. H. NeumanN

F. WOLF

K. YosHidA

\section{SUPPORTING INSTITUTIONS}

UNIVERSITY OF BRITISH COLUMBIA UNIVERSITY OF SOUTHERN CALIFONIA CALIFORNIA INSTITUTE OF TECHNOLOGY UNIVERSITY OF CALIFORNIA MONTANA STATE UNIVERSITY STANFORD UNIVERSITY UNIVERSITY OF HAWAII UNIVERSITY OF NEVADA, RENO UNIVERSITY OF TOKYO U'NIVERSITY OF UTAH NEW MEXICO STATE UNIVERSITY WASHINGTON STATE UNIVERSITY OREGON STATE UNIVERSITY UNIVERSITY OF OREGON UNIVERSITY OF WASHINGTON 


\section{Pacific Journal of Mathematics}

\section{Vol. 90, No. $1 \quad$ September, 1980}

Shashi Prabha Arya and M. K. Singal, On the locally countable sum

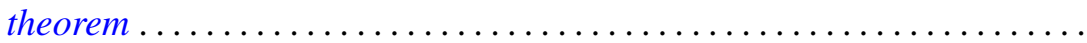

John Theodore Baldwin and David William Kueker, Ramsey quantifiers and

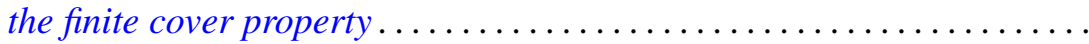

Richard Body and Roy Rene Douglas, Unique factorization of rational

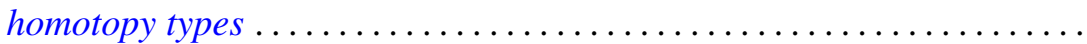

Ethan Bolker and Ben G. Roth, When is a bipartite graph a rigid

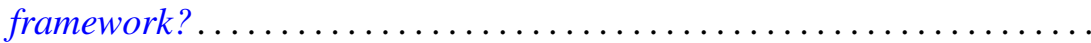

Alicia B. Winslow, Continua in the Stone-Čech remainder of $R^{2} \ldots \ldots \ldots$

Richard D. Carmichael and Elmer Kinji Hayashi, Analytic functions in tubes which are representable by Fourier-Laplace integrals ..............

Stephen D. Cohen, The Galois group of a polynomial with two indeterminate coefficients ..............................

Russell Allan Johnson, Strong liftings commuting with minimal distal

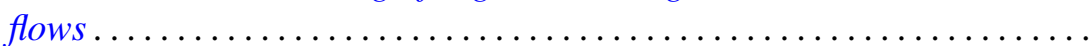

Elgin Harold Johnston, The boundary modulus of continuity of harmonic

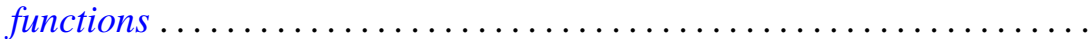

Akio Kawauchi and Takao Matumoto, An estimate of infinite cyclic

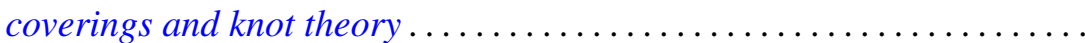

Keith Milo Kendig, Moiré phenomena in algebraic geometry: rational alternations in $\mathbf{R}^{2}$...

Roger T. Lewis and Lynne C. Wright, Comparison and oscillation criteria for selfadjoint vector-matrix differential equations .

Teck Cheong Lim, Asymptotic centers and nonexpansive mappings in conjugate Banach spaces .......................

David John Lutzer and Robert Allen McCoy, Category in function spaces.

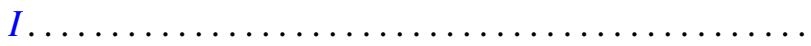

Richard A. Mollin, Induced p-elements in the Schur group ...

Jonathan Simon, Wirtinger approximations and the knot groups of $F^{n}$ in $S^{n+2}$

Robert L. Snider, The zero divisor conjecture for some solvable groups...

H. M. (Hari Mohan) Srivastava, A note on the Konhauser sets of biorthogonal polynomials suggested by the Laguerre polynomials...

Nicholas Th. Varopoulos, A probabilistic proof of the Garnett-Jones theorem on BMO.

Frank Arvey Wattenberg, $[0, \infty]$-valued, translation invariant measures on $N$ and the Dedekind completion of ${ }^{*} R \ldots \ldots \ldots \ldots . .$. 